15

\title{
Малогабаритный многоканальный анализатор энергии заряженных частиц на основе конусного разрезанного электрода
}

\author{
(C) Т.Я. Фишкова
}

Физико- технический институт им. А.Ф. Иофрфе РАН, 194021 Санкт-Петербург, Россия

e-mail: fishkova@mail.ru

Поступило в Редакцию 6 августа 2018 г.

В окончательной редакции 7 ноября 2018 г.

Принято к публикации 25 ноября 2018 г.

Предложен и рассчитан электростатический малогабаритный анализатор для получения энергетического спектра, с возможностью одновременной (параллельной) регистрации заряженных частиц в широком диапазоне их энергий, что, особенно, важно при быстропротекающих процессах в различного рода исследуемых веществах. Энергоанализатор состоит из внешнего разрезанного на части электрода конической формы с закрытыми торцами, питание которого осуществляется по закону нечетных чисел, и заземленного внутреннего цилиндрического электрода, на поверхность которого фокусируются пучки заряженных частиц в широком диапазоне энергий. Для повышения остроты фокусировки пучков низких энергий введен дополнительный конусный электрод очень малых размеров. Несмотря на малую величину коэффициента дисперсии по энергии, найден оптимальный режим работы предложенного анализатора с разрешением по энергии не хуже $1 \%$.

DOI: $10.21883 /$ JTF.2019.05.47487.300-18

Дисперсия анализаторов заряженных частиц по энергии зависит от протяженности их траекторий в электростатическом поле. Поэтому уменьшение габаритов анализатора ведет к ухудшению его разрешения по энергии. Задача настоящей работы состоит в отыскании и исследовании многоканального электростатического анализатора как можно меньших размеров с хорошим разрешением по энергии.

Решение поставленной задачи осуществляется за счет использования в электростатическом анализаторе (из двух коаксиальных электродов) внешнего конусного разрезанного электрода с упрощенным питанием на его частях. Схема предлагаемого многоканального светосильного электростатического энергоанализатора с ходом траекторий заряженных частиц в широком диапазоне их энергий представлена на рис. 1. Как и в последних работах автора [1,2], посвященных расчету параметров запатентованной ранее модели энергоанализатора, предлагаемый здесь анализатор состоит из двух коаксиальных электродов с закрытыми торцами. Внутренний электрод выполнен в виде цилиндра радиуса $R$, a внешний - в виде разрезанного на части конуса с начальным радиусом $R_{0}$. Следует отметить, что с точки зрения конструкции прибора логично использовать в качестве внешнего электрода именно конус, так как в него хорошо вписываются пучки заряженных частиц по мере роста их энергий.

Выбор оптимальной геометрии многоканального энергоанализатора проводился численно по программе ТЕО для осесимметричных систем. Варьировались пять геометрических параметров: угол раствора конусного электрода, количество частей, на которое он разрезан, и его начальный радиус, а также положение исследуемого объекта, расположенного вне электростатического поля, и угол входа центральной траектории пучка заряженных частиц. При этом способ питания разрезанного конусного электрода и величины потенциалов на его частях менялись либо по ранее часто используемому линейному закону, либо значительно проще - по предлагаемому в настоящей работе закону нечетных чисел.

В результате численных расчетов выявлена оптимальная геометрия и способ питания предлагаемого многоканального энергоанализатора, которые приводятся ниже. Конусный электрод 1 с углом полураствора $\beta=10^{\circ}$, величиной малого радиуса $R_{0}=2.5 \mathrm{R}$ и длиной $L=10.5 \mathrm{R}$ (рис. $1, a)$ разрезан на шесть равных частей размером $l_{n}=1.5 \mathrm{R}$, расположенных на расстояниях $s_{n}=0.25 \mathrm{R}$ друг от друга. Внутренний цилиндрический электрод 2 радиуса $R$ соединен с торцевым плоским электродом 3 , через диафрагму в котором происходит впуск пучков частиц различных энергий. Задний плоский торцевой электрод 4 расположен на малом расстоянии от торцов конусного и цилиндрического электродов, не допускающем электрического пробоя. Такая конструкция имеет значительно меньшие размеры по сравнению с аналогичными многоканальными энергоанализаторами с разрезанными плоскими, либо с цилиндрическими электродами, исследованными ранее. Так, размеры предлагаемого многоканального анализатора в два раза меньше в продольном и поперечном направлениях по сравнению с исследованным ранее автором в работах $[1,2]$.

Питание разрезанного конусного электрода осуществлялось по новому. Ранее в различного рода многоканальных энергоанализаторах напряжения на разрезанном 

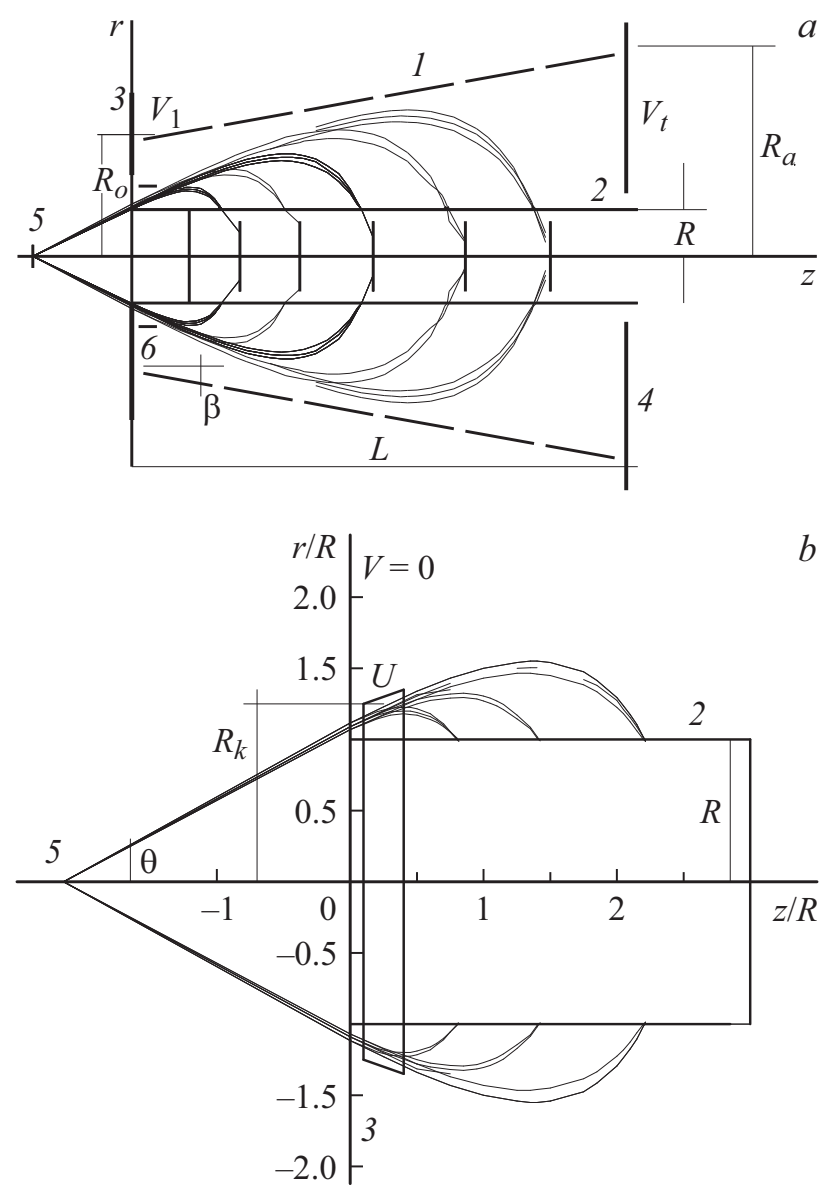

Рис. 1. Схема малогабаритного многоканального энергоанализатора с ходом траекторий заряженных частиц в диапазоне средних и высоких энергий $E=(10-50) E_{\min }(a)$ и ход траекторий пучков низких энергий вблизи малого дополнительного конического электрода при $E=E_{\min }, E=2.5 E_{\min }, E=5 E_{\min }(b)$.

электроде плоской, либо цилиндрической формы задавалось главным образом по линейному закону, реже по закону $3 / 2$, либо по квадратичному закону. Расчеты, выполненные в настоящей работе, показали, что потенциалы на частях конусного разрезанного электрода можно задавать проще - по закону нечетных чисел:

$$
V_{n}=V_{1}(2 n-1),
$$

где $V_{1}$ - потенциал на первой части разрезного конуса, $n-$ порядковый номер части разрезного конуса, отсчитываемый от входного торцевого электрода. Кроме того, расчеты показали, что оптимальная величина потенциала заднего плоского торцевого электрода $V_{t}$ должна быть равна последнему члену ряда (1). Поскольку в оптимальной системе конусный электрод разрезан на шесть частей, то $n=7$, поэтому $V_{t}=13 V_{1}$. Внутренний цилиндрический электрод вместе с передним торцевым плоским электродом как это обычно бывает для такого рода систем заземлен.

Одновременная фокусировка пучков в широком диапазоне энергий осуществлялась на поверхность внутренне- го цилиндрического электрода 2 , который снабжен кольцевыми щелями, затянутыми металлической сеткой для прохождения пучков заряженных частиц. Пройдя щели, пучок попадает в детектор в виде плоских электродов, установленных перпендикулярно продольной оси системы, которые подключены к многоканальному измерительному устройству.

Расчеты выявили оптимальный режим работы, при котором центральная траектория пучков различных энергий, исходящая из центра образца под углами $\theta=26.7^{\circ}$, фокусируются в диапазоне энергий $E=(1-50) E_{\min }$ на внутренний цилиндр при положении исследуемого образца 5 на расстоянии от входа в поле, равном $z_{i}=2.2 \mathrm{R}$. Напряжение на первой части разрезного конуса должно быть равно $V_{1}=4.4\left(E_{\min } / e\right)$, где $E_{\min }-$ минимальная энергия, $e-$ заряд рабочих частиц, напряжения на последующих частях подаются согласно закону нечетных чисел (1). При этом положение точек фокусировки центральных траекторий пучков различных энергий на поверхности цилиндрического электрода $z_{f} / R$, a также коэффициент линейной дисперсии по энергии $D / R$ малогабаритного энергоанализатора представлены на рис. 2. Видно, что расстояние от входа пучков до точек фокусировки в поле растет практически по линейному закону. Дисперсия анализатора по энергии также растет в широкой области энергий $E \leq 35 E_{\min }$, затем практически не меняется, а в области энергий, близких к максимальной, $E=(0.9-1.0) E_{\max }$ немного уменьшается из-за близости заднего торцевого электрода с наибольшим питающим напряжением. Однако последнее обстоятельство способствует улучшению остроты фокусировки в этой области.

Для пучков низких энергий степень фокусировки недостаточна, так как очень мала величина коэффициен-

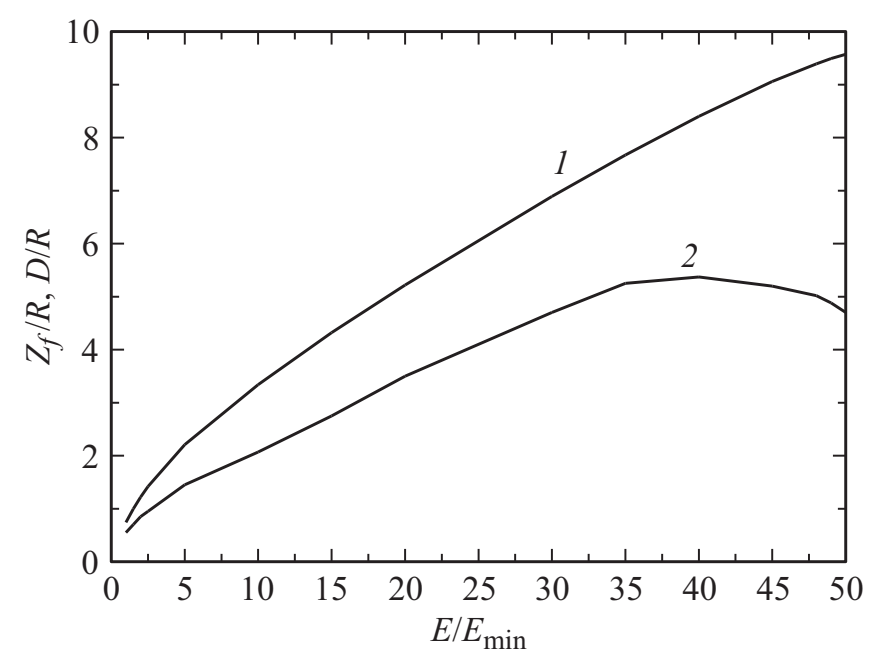

Рис. 2. Параметры малогабаритного энергоанализатора в широком диапазоне одновременно регистрируемых энергий: кривая $1-z_{f} / R$ (расстояние от входа в поле до точки фокусировки центральной траектории пучков заряженных частиц различных энергий), $2-D / R$ (коэффициент линейной дисперсии системы по энергии). 
та линейной дисперсии, что не позволяет получить достаточное разрешение по энергии. Поэтому для улучшения фокусировки в этой области введен дополнительный короткий конусный электрод 6 с таким же углом полураствора, как и у основного разрезного конуса $\beta=10^{\circ}$. Варьировалась его геометрия и питание. Оптимальными для работы дополнительного конуса оказались его положение на расстоянии $0.1 R$ от переднего плоского торца, начальный радиус $R_{k}=1.25 \mathrm{R}$, длина $l=0.2 \mathrm{R}$ и питающий потенциал $U=0.13 V_{1}$. Отметим, что в областях средних и высоких энергий дополнительный электрод влияния не оказывает.

Поскольку в оптимальном режиме работы центральная траектория пучков входит в поле под углом $\theta=26.7^{\circ}$, то при угле раствора пучка $\alpha= \pm 0.5^{\circ}$ размер пучка, фокусированного на внутренний цилиндрический электрод, в области средних и высоких энергий увеличивается с ростом энергии пучка и составляет $(1-4) \cdot 10^{-2} \mathrm{R}$. В этих областях с ростом энергии пучка соответственно растет и коэффициент линейной дисперсии (рис. 2). Размеры пятна для частиц низких энергий, сфокусированного с участием дополнительного конусного электрода, значительно меньше и составляют $(3-6) \cdot 10^{-3} \mathrm{R}$, однако, поскольку дисперсия на низких энергиях мала, то разрешение во всем диапазоне энергий различается незначительно.

Разрешение анализатора по энергии $\rho=\delta E / E$ является его основным параметром. Для исследованного в работе многоканального малогабаритного анализатора в широкой области изменения энергии пучка $E=(1-50) E_{\min }$ разрешение оказалось равно $\rho=(4-9) \cdot 10^{-3}$. При этом из-за наличия в системе осевой симметрии светосила $\Omega$ рассматриваемого энергоанализатора при угле раствора пучков $\alpha=1^{\circ}$ составляет $\Omega=0.39 \%$ от $4 \pi$, т. е. аналогична энергоанализаторам $[1,2]$ и почти на два порядка превышает светосилу многоканальных энергоанализаторов из плоских электродов.

Подводя итог проведенной работы, отметим, что предложен и численно рассчитан малогабаритный светосильный анализатор энергии заряженных частиц с широким диапазоном одновременно регистрируемых энергий, состоящий из двух коаксиальных электродов с закрытыми торцами - цилиндрического и разрезанного на части конического. Найдены оптимальные геометрические размеры системы и использовано питание разрезного конусного электрода по нетрадиционному для многоканальных электростатических анализаторов закону нечетных чисел. Разрешение предложенного многоканального энергоанализатора оказалось не хуже $1 \%$.

\section{Список литературы}

[1] Фишкова Т.Я. // ЖТФ. 2018. Т. 88. Вып. 1. С. 117-120.

[2] Фишкова Т.Я. // ЖТФ. 2018. Т. 88. Вып. 7. С. 1094-1097. 